\title{
Patrón espacial y métodos de muestreo para Bertholletia excelsa (castaña de Pará) en la Mesorregión del Bajo Amazonas, estado de Pará, Brasil
}

\author{
Spatial pattern and sampling methods for Brazil nut tree in the mesoregion \\ of the Lower Amazon, state of Para, Brazil
}

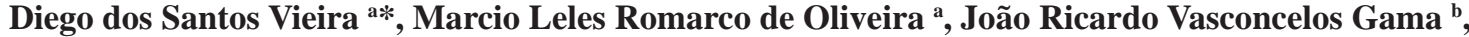 \\ Evandro Luiz Mendonça Machado a, Eric Bastos Görgens a \\ *Autor de correspondencia: ${ }^{\text {a }}$ Federal University of Jequitinhonha and Mucuri Valleys, Department of Forestry, \\ Diamantina, Brazil, tel.: 55-93-991973755, diegovieir4@gmail.com \\ ${ }^{\mathrm{b}}$ Federal University of Para West, Department of Forestry, Santarém, Brazil.
}

\section{SUMMARY}

The aim of this study was to analyze the spatial pattern and sampling methods for Bertholletia excelsa. The inventory of exploration, by mapping Cartesian coordinates of all trees with $\mathrm{dbh} \geq 20 \mathrm{~cm}$, was carried out in an area of 1,000.31 ha. For the definition of the diametric structure, multivariate techniques were used: cluster and discriminant analysis. To analyze the spatial patterns, individuals were grouped into three levels: population, young individuals and adults; being the random deviation defined by the Ripley's K function. The forest inventories were simulated with a simple random sampling, systematic and adaptive, taking into account plots of 2,500 $\mathrm{m}^{2}$, with a sampling intensity of $15 \%$ and an error range of $10 \%$. Comparisons between sampling methods were performed for the accuracy and precision. Four hundred and forty six trees were registered, of which 59 were young individuals and 387 were adults. A low number of trees in the early stages and a high number of individuals in the intermediate classes characterize the diametric structure. The spatial pattern of the adult population was aggregated, while the young individuals were randomly distributed. Systematic sampling is the best procedure to estimate the total number of individuals of $B$. excelsa; however, there is a need to investigate the effect of aggregation and size of the largest plots of 0.25 hectares on the estimates of adaptive cluster sampling.

Key words: adaptive cluster sampling, Ripley's K function, Amazon.

\section{RESUMEN}

El objetivo de este estudio fue analizar el patrón espacial y los métodos de muestreo para Bertholletia excelsa. El inventario de exploración, con mapeo de coordenadas cartesianas de los árboles con dap $\geq 20$ cm, se llevó a cabo en un área de 1.000,31 ha. Para la definición de la estructura diamétrica se utilizaron técnicas multivariadas: análisis de conglomerado y discriminante. Para analizar los patrones espaciales, los individuos fueron agrupados en tres niveles: población, jóvenes y adultos, siendo la desviación de aleatoriedad definida por la función K de Ripley. Fueron simulados los inventarios forestales con el muestreo aleatorio simple, sistemático y adaptativo, teniendo en cuenta las parcelas de $2.500 \mathrm{~m}^{2}$, con una intensidad de muestreo del $15 \%$ y un margen de error de $10 \%$. Las comparaciones entre los métodos de muestreo se realizaron por exactitud y precisión. Se registraron 446 árboles, de los cuales 59 individuos eran jóvenes y 387 eran adultos. La estructura diamétrica se caracterizó por un bajo número de árboles en los primeros grados y un alto número de individuos en las clases intermedias. El patrón espacial de la población y de los adultos fue agregado, mientras que los menores fueron distribuidos al azar. El muestreo sistemático fue el mejor procedimiento para estimar el número total de individuos de B. excelsa, sin embargo, hay necesidad de investigar el efecto de agregación y el tamaño de las parcelas mayores de $2.500 \mathrm{~m}^{2}$ sobre los estimadores de muestreo adaptativo por conglomerados.

Palabras clave: muestreo adaptativo por conglomerados, función K de Ripley, Amazonas.

\section{INTRODUCCIÓN}

La valoración de los recursos forestales, limitados a la producción de madera, ha sido modificada en el escenario macroeconómico, llegando a ser cada vez más presente el interés en otros productos y beneficios (Bruzinga et al. 2013). Los productos forestales no madereros son, alter- nativamente, uno de los grupos más prometedores desde el punto de vista del mercado, gracias a su cantidad, su versatilidad, su variedad de usos y su diferenciación de otros productos básicos. Entre las especies de valor en la Amazonía, que se caracterizan por no tener gran potencial maderable, destaca Bertholletia excelsa Bonpl. (castaña de Pará), por su importancia social, ecológi- 
ca y económica por su fruto para la región amazónica (Salomão 2009).

La castaña de Pará es uno de los productos forestales no madereros más conocidos y consolidados en los mercados nacional y de exportación, constituyendo la única colecta de semillas en cultivos individuales que se venden en mercados extranjeros, realizada casi exclusivamente en los bosques tropicales primarios (Peres et al. 2003). Además, es una especie clave para el desarrollo socioeconómico de las comunidades y el mantenimiento de los beneficios directos e indirectos de los bosques (Tonini 2013). Sin embargo, la gestión del bosque $B$. excelsa en la Amazonia oriental no es todavía una realidad, dándose la recolección de semillas sin un previo desarrollo de un plan de manejo forestal sostenible.

La preparación de un plan de manejo forestal sostenible es fundamental para lograr el censo forestal de las especies a ser administradas, con el fin de obtener una estimación fiable del número de árboles por especie y el seguimiento del origen de los productos con el fin de tener una mayor transparencia de las actividades y facilitar la inspección de las agencias ambientales y certificadoras (Alechandre et al. 2007). Sin embargo, inspeccionar a todos los individuos en el área de gestión no es factible debido al tiempo y los recursos limitados, lo que hace necesario el uso de metodologías de muestreo precisas, representativas y económicamente viables (Tonini 2013)

El interés del uso y el comercio de este producto crece junto con la necesidad de investigar sobre la cuantificación de las especies, dado que hay pocos estudios que tratan de definir los sistemas de muestreo para las especies no maderables en la Amazonia (Wong et al. 2001). Las características comunes a las especies no maderables, tales como la estacionalidad, la movilidad, la necesidad de cuantificar mediante métodos no destructivos, y especialmente, su rareza y agrupamiento, dificultan la utilización de técnicas tradicionales de muestreo (Vieira 2015). Sin embargo, Thompson (1990) y Thompson y Seber (1996) introdujeron una metodología de muestreo especializada para las poblaciones raras y agrupadas, denominada muestreo por conglomerado adaptativo. La técnica utiliza la información sobre los valores observados para tener más éxito en la recopilación de las unidades de una población rara, lo que aumenta la eficiencia de estimación, pues se espera que sea más probable encontrar un elemento raro en la proximidad del otro elemento cuando la población es agregada.

Según Christman (2000) y Smith et al. (2004), el muestreo adaptativo por conglomerado es una de las mejores técnicas para ser aplicada en el contexto de las poblaciones raras y agrupadas. Por lo tanto, teniendo en cuenta la rareza de las características y la agregación de las especies no maderables, el objetivo de este estudio es evaluar el patrón espacial y los métodos de muestreo para estimar el número de árboles por hectárea de una población de $B$. excelsa en la mesorregión del Bajo Amazonas en el estado de Pará, Brasil. La principal hipótesis que se plantea es que el muestreo adaptativo por conglomerados es el mejor procedimiento para estimar el número total de individuos de B. excelsa. Adicionalmente, se espera que el patrón espacial de la especie sea agregado debido su síndrome de dispersión por medio de la gravedad.

\section{METODOS}

Área de estudio. El estudio se realizó en la unidad de producción anual $N^{\circ}$ 8, en la Unidad de Manejo Florestal Samambaia (1.000,31 ha), que pertenece a la Cooperativa Mixta de la Floresta Nacional de Tapajós (COOMFLONA). El clima de la región, según la clasificación de Köppen, corresponde al tipo Ami, con una temperatura y una precipitación anual de $25,5{ }^{\circ} \mathrm{C}$ y $1.820 \mathrm{~mm}$, respectivamente. El relieve es ligeramente accidentado y presenta una topografía que van desde suaves ondulaciones a ondulada, con un predominio de Latosol Amarillo Distrófico. La vegetación está clasificada como Bosque Ombrófilo Denso de Tierra Firme, que se caracteriza por el predominio de los grandes individuos arbóreos y la abundancia de lianas leñosas, palmas y epífitas (Veloso et al. 1991).

Recolección de datos. Se realizó un inventario, con el mapeo de los individuos en coordenadas cartesianas $(\mathrm{X}, \mathrm{Y})$. La unidad de producción anual se dividió en diez unidades de trabajo de 100 hectáreas cada una (1.000 x 1.000 m). Para facilitar la numeración y el mapeo de los representantes de las especies en cada unidad de trabajo, se abrieron cortes paralelos en la dirección norte-sur, distantes por $50 \mathrm{~m}$ entre sí. En los cortes fueron colocados marcadores cada $25 \mathrm{~m}$, con su respectiva medida en relación al origen, para efectuar el posterior registro de coordenadas de cada árbol. La coordenada $\mathrm{X}$ de cada individuo se obtuvo a través del número del corte y la distancia de cada árbol en relación a ella.

Aunque el inventario ha considerado todas las especies comerciales con un diámetro de 1,30 m por encima del suelo (dap) mayor o igual a $20 \mathrm{~cm}$, se seleccionó la especie B. excelsa. Contando con las coordenadas de todos los individuos, se produjo un mapa dividido en 4.000 unidades de la muestra con $2.500 \mathrm{~m}^{2}$ (0,25 ha) de cada área, utilizando las muestras de las simulaciones. La identificación de la presencia de árboles de la especie en las muestras se realizó utilizando las coordenadas X e Y de los árboles con los límites de cada unidad de la muestra.

Distribución diamétrica. Para determinar la estructura diamétrica de la comunidad, se adaptó el procedimiento propuesto por Souza et al. (2003), utilizado para establecer los estratos de altura total. El uso de este método ocurrió debido a la falta de criterios para la determinación de la amplitud de las clases dimétrica, además de ser una nueva forma de evaluar la distribución del diámetro. Inicialmente se organizaron, en orden creciente de diámetro, todos los individuos clasificados en las clases con una amplitud de 
$1 \mathrm{~cm}$. Posteriormente se elaboró una matriz D de datos del diámetro, donde cada variable de esta matriz representa el diámetro del árbol $i$-ésimo en la clase de diámetro $j$-ésimo. La matriz D fue la entrada al análisis de conglomerados y discriminante. La distancia euclidiana y el método de Ward se utilizaron para definir los grupos constituidos por clases de $1 \mathrm{~cm}$ de diámetro, llamadas clases diamétricas. Sobre el dendrograma resultante de este análisis fue trazada una línea de corte, a fin de elaborar una serie coherente de clases diamétricas. Para hacer la distinción y la clasificación de las clases diamétricas, se utilizó el análisis discriminante.

Se probaron seis funciones para la densidad de probabilidad para la obtención de las distribuciones de frecuencia de los árboles en cada clase de diámetro. Las funciones correspondientes a distribuciones ajustadas fueron Weibull2P, Weibull-3P, Gama, Normal, Logística, Lognormal. Los parámetros de las funciones Weibull-2P y Weibull-3P fueron obtenidos por el método de máxima verosimilitud, mientras que los demás fueron ajustados por el método de los momentos. Para evaluar la calidad del ajuste, se utilizó la adhesión de Kolmogorov-Smirnov (K-S), en el 99 \% de probabilidad.

Rareza numérica y patrón espacial. Como criterio de rareza numérica, se consideró que si la especie posee un individuo por hectárea sería considerada una especie rara (Soares et al. 2009). Para definir el patrón espacial fue seleccionada la función K(s) de Ripley (1979), aplicada a la población, y dos subgrupos demográficos: los adultos reproductivamente maduros (dap $\geq 50 \mathrm{~cm}$ ) y los jóvenes (dap < $50 \mathrm{~cm}$ ) (Wadt et al. 2005). De una manera simplificada, se define el radio de un círculo de $5 \mathrm{~m}$ centrado en cada árbol, donde se contó el número de vecinos presentes en el área de este círculo. Variando el radio $s$ a una distancia máxima de 2.000 metros, se detectó el patrón espacial de la especie en diferentes escalas de distancia.

Se produjeron casos especiales en que los árboles estaban cerca del borde de la zona, ya que, como la función $\mathrm{K}(s)$ es acumulativa y son calculadas todas las distancias entre todos los puntos, los árboles cercanos con un radio mayor al límite del mapa no podían ser interpretados como si no tuvieran vecinos. Los vecinos existían, pero por estar fuera de los límites de la zona no fueron contados. Por lo tanto, el número de árboles vecinos de los árboles cerca de los límites del mapa sería menor que para el otro, causando un sesgo en el estimador de la función K(s). Por consiguiente, se utilizó un estimador de la función $\mathrm{K}(\mathrm{s})$ con la corrección del límite isotrópico, conforme a lo descrito por Ripley (1979) [1].

$\hat{K}(s)=\frac{1}{\lambda n} \sum_{i=1}^{n} \sum_{i=1}^{n} W_{1}^{-1}\left(X_{i}, X_{j}\right) l\left(\left\|X_{i}-X_{j}\right\| \leq s\right)$ para $i \neq j$ e $s>0$

donde $n$ = número de árboles en la región de estudio; $x_{i} \mathrm{e}$ $x_{j}=$ coordenadas de los puntos del mapa; $\left\|x_{i}-x_{j}\right\|$ distancia euclidiana entre localizadores $x_{i}$ e $x_{i}$; $s=$ vector arbitrario de distancias; $\hat{\lambda}_{n}=\mathrm{n} /\|A\|=$ número de árboles dividido por el área de la región de estudio, siendo un estimador no sesgado de la intensidad del proceso; $\mathrm{W}_{\mathrm{I}}\left(x_{i}, x_{j}\right)=$ función de corrección del límite isotrópico, que representa la proporción de la circunferencia con su centro en $x_{i}$ y con radio $\left\|x_{i}-x_{j}\right\|$ que está fuera de cualquier polígono convexo; y $\mathrm{I}(\mathrm{U})$ = función indicadora que asume el valor 1 cuando la condición U es verdadera y cero cuando es falsa.

Para analizar los datos de forma gráfica, fueron construidos intervalos (envelopes) de confianza mediante 1.000 simulaciones de Monte Carlo, realizadas por medio del modelo de Completa Aleatoriedad Espacial (CAE). Luego, se calculó la función K(s) para los resultados de las simulaciones, almacenándose los valores mínimos y máximos de la estimación de $\mathrm{K}(s)$ que se utiliza para generar intervalos de confianza con una probabilidad de 99 $\%$. Para facilitar el análisis, los valores de la función $\mathrm{K}(s)$ fueron transformados a L(s) [2] y se distribuyen de forma gráfica donde los ejes de abscisas y ordenadas representan respectivamente, las distancias s acumuladas y los valores transformados de la función de $\mathrm{K}(\mathrm{s})$.

$$
\hat{L}(s)=\sqrt{\frac{\hat{K}(s)}{\pi}}-s
$$

Como resultado, se obtuvieron los intervalos de confianza identificadas por dos líneas punteadas, una positiva y una negativa. Si los valores observados de L(s) están dentro del intervalo construido, el patrón espacial es aleatorio, de lo contrario se rechaza la hipótesis nula de completa aleatoriedad espacial y se asume que el patrón espacial de los individuos es agregado cuando pasa por encima del límite superior del intervalo, y regular, al pasar el límite inferior (Ripley 1979).

Procedimiento de muestreo. Se utilizaron tres procedimientos de muestreo: muestreo aleatorio simple sin reemplazo de unidades de muestra; muestreo sistemático con aleatoriedad solamente en el primer tramo y los demás distribuidos de manera equidistante; y el muestreo adaptativo por conglomerado, con inclusión de la condición determinada por la presencia de al menos un individuo $(y i \geq 1)$ de la especie (Thompson y Seber 1996). Para todas las alternativas se utilizó una intensidad de muestra de $15 \%$.

En el muestreo adaptativo por conglomerado fueron simulados cuatro procedimientos diferentes: el muestreo adaptativo por conglomerados tradicional por precariedad (MAC-T); muestreo adaptativo por conglomerados tradicionales en bandas (MAC-TF), muestreo adaptativo por conglomerados modificados por precariedad (MAC-M) y muestras de adaptación de conglomerados modificados en bandas (MAC-MF). Se entiende por "conglomerado tradicional” al conjunto de parcelas constituido por una unidad de red y cuatro unidades fronterizas dispuestas en el norte, sur, este y oeste; "conglomerados modificados" como un 
conjunto constituido por un conglomerado tradicional más cuatro unidades borde situadas en las diagonales de la unidad de red; y "por precariedad" y "en banda” como el modo en que las unidades de red fueron simuladas (figura 1). En estos métodos de muestreo, las unidades de muestreo que no cumplían con los criterios de inclusión fueron consideradas un conglomerado.

Para MAC-T y MAC-M fueron utilizadas como muestras iniciales las mismas unidades seleccionadas del muestreo aleatorio simple y muestreo sistemático (Soares et al. 2009), mientras que los grupos de MAC-TF y MAC-MF se simularon en bandas distribuidas sistemáticamente en la unidad de producción anual. Las bandas fueron simuladas considerando dos caminos presentes en la zona que divide la unidad de producción anual en dos transectas de 1.000 x $3.000 \mathrm{~m}$ y 1.000 x $4.000 \mathrm{~m}$, dispuestas en paralelo. En cada uno de los transectas las bandas fueron simuladas a 250 y $750 \mathrm{~m}$ del margen de la carretera con una longitud relacionada al tamaño de cada transecta.

En muestreo aleatorio simple y muestreo sistemático se distribuyeron 600 unidades de muestreo en toda la zona, dispuestas en un sentido de norte a sur. Para las alternativas de muestreo aleatorio simple, muestreo sistemático, MACTy MAC-M se realizaron 30 simulaciones (Cochran 1977), mientras que MAC-TF y MAC-MF se simularon una sola vez, ya que se refieren a los procedimientos para llevar a cabo la búsqueda de los individuos de la especie en las bandas simuladas, de esta manera las estimaciones de la media para todas las simulaciones fueron iguales.
Análisis de muestras. Independientemente del procedimiento de muestreo, se calcularon para cada simulación las estimaciones promedio del número de individuos por hectárea, la varianza media, error estándar de la media de error de muestreo, los intervalos de confianza y precisión. En la estimación de la media y la varianza de la media de muestreo aleatorio simple y muestreo sistemático se utilizaron estimadores comúnmente utilizados y descritos por Shiver y Borders (1996). Con el fin de cuantificar la intensidad de muestreo necesario para obtener errores menores que el $10 \%$ se calculó para los métodos muestreo aleatorio simple e muestreo sistemático o dimensionamiento de la muestra (Shiver y Borders 1996). Para el muestreo adaptativo por conglomerado, el promedio de las estimaciones [3] y la varianza media [4] fueron obtenidos por los estimadores modificados de Hansen-Hurwitz (Hansen y Hurwitz 1943, Thompson 1990), calculados por las siguientes expresiones:

$$
\begin{gathered}
\bar{Y}_{H H^{*}}=\left(\frac{1}{n_{1}} \sum_{i=1}^{n_{1}} w_{i}\right), \text { sendo } w_{i}=\frac{\sum_{i=1}^{m} y_{i}}{m_{i}} \\
S_{\bar{Y}_{H H^{*}}}^{2}=\frac{N-n_{1}}{N n_{i}\left(n_{i}-1\right)} \sum_{i=1}^{n_{1}}\left(w_{i}-\bar{Y}_{H H^{*}}\right)^{2}
\end{gathered}
$$

en donde: $\bar{Y}_{H H^{*}}=$ estimador de Hansen-Hurwitz modificado para la media de individuos por unidad de muestra; $S_{\bar{Y}_{H H^{*}}}^{2}=$ estimador de Hansen-Hurwitz modificada para la varianza
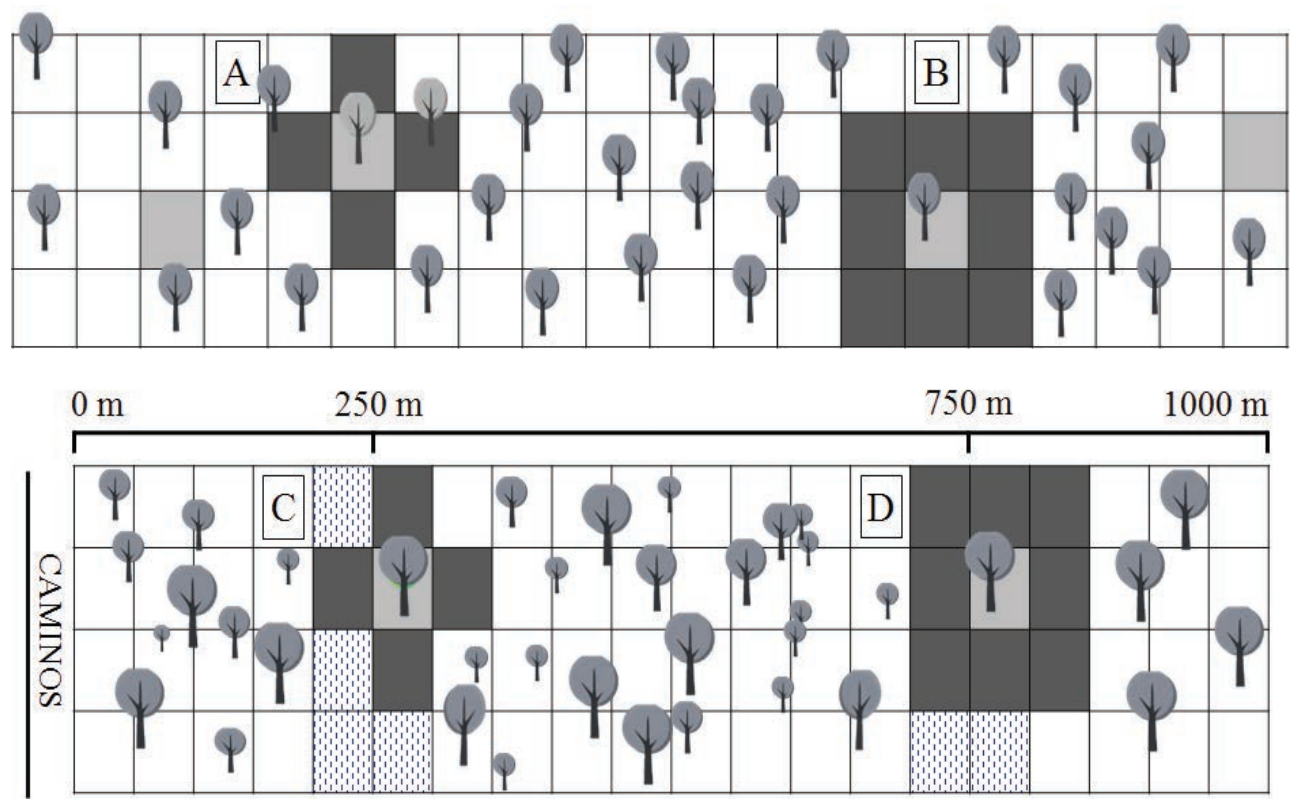

Figura 1. Ilustración de los conglomerados. $A$ = conglomerado tradicional por precariedad; $B$ = conglomerado modificado por precariedad; $\mathrm{C}=$ conglomerado tradicional en bandas; $\mathrm{D}=$ conglomerado modificado en bandas.

Illustration of the conglomerates. $\mathrm{A}=$ traditional conglomerate by randomization; $\mathrm{B}=$ modified conglomerate by randomization; $\mathrm{C}=$ traditional conglomerate in bands; $\mathrm{D}=$ modified conglomerate in bands. 
media; $N$ = número total de unidades de la muestra en la población; $n_{i}=$ número inicial de unidades de la muestra; $m_{i}=$ número de unidades de red; $w_{i}=$ media de las $m_{i}$ observaciones de la $i$-ésima red; e $y i$ = número de individuos de la especies en la $i$-ésima unidad de muestra.

La comparación entre el muestreo estimado se realizó por medio de la precisión y exactitud. Los cálculos se realizaron utilizando el software $\mathrm{R}$ versión 3.1.2, siendo la función $\mathrm{K}(\mathrm{s})$ estimada a través del paquete Splancs.

\section{RESULTADOS}

Estructura diamétrica y espacial. En la encuesta de prospección se registraron 446 individuos $\left(0,446\right.$ árboles ha $\left.{ }^{-1}\right)$ de los cuales 59 (0,059 árboles ha-1) son individuos jóvenes en edad no reproductiva y 387 ( 0,387 árboles hä $\left.{ }^{-1}\right)$ son adultos reproductivamente maduros. Los daps mínimo y máximo muestreados fueron de 23,9 a 190,9 cm, respectivamente, para un diámetro medio de 79,6 $\pm 25,1 \mathrm{~cm}$. Teniendo en cuenta el dendrograma con la línea de corte a nivel de homogeneidad del $50 \%$, fueron registrados 22 grupos homogéneos y distintos (clases diamétricas), con una amplitud de 7,9 cm para las 20 clases iniciales y 3,9 y 4,1 cm para las dos últimas clases, respectivamente (figura 2). La disposición de los árboles individuales con amplitud de $1 \mathrm{~cm}$ permitió agrupar secuencialmente, en orden ascendente, las clases diametrales. El uso del análisis discriminante entre las clases diamétricas obtenidas en el análisis de conglomerados mostró diferencias significativas $(F=21,147)$, con valores estadísticos Wilk's Lambda cercanos a cero, evidenciando que el $100 \%$ de las clases diamétricas fueron clasificadas correctamente.
A partir del análisis visual del histograma de frecuencias, se observó que la especie tiene un bajo número de individuos en las clases diamétricas más bajas y elevada frecuencia en las clases intermedias, con una reducción significativa en dirección a las mayores clases diamétricas (figura 2). Desde un punto de vista estadístico, este comportamiento puede describirse por las funciones de Weibull-2P $\left(\mathrm{D}_{\text {calc }}=0,036 ; \mathrm{D}_{\text {tab }}=0,076\right)$, Gama $\left(\mathrm{D}_{\text {calc }}=0,038\right.$; $\left.\mathrm{D}_{\text {tab }}=0,076\right)$, Normal $\left(\mathrm{D}_{\text {calc }}=0,046 ; \mathrm{D}_{\text {tab }}=0,076\right)$ e Weibull-3P ( $\left.\mathrm{D}_{\text {calc }}=0,055 ; \mathrm{D}_{\text {tab }}=0,076\right)$, que fueron estadísticamente similares a la distribución observada. Por otra parte, la función normal logarítmica $\left(\mathrm{D}_{\text {calc }}=0,138 ; \mathrm{D}_{\mathrm{tab}}=0,076\right)$ se demostró inadecuada para describir la distribución del diámetro de la población. Para tomar la decisión de elegir la mejor función, fueron trazadas las curvas de frecuencia estimadas sobre los histogramas de las frecuencias observadas, que permitirán constatar la tendencia a subestimar el número de árboles en las clases diamétricas intermedias.

Sobre la base de la aplicación de la función $\mathrm{K}(\mathrm{s})$ de Ripley, se encontró que el patrón espacial de la población no cumple con la hipótesis nula de completa aleatoriedad espacial, lo que indica que hay un patrón espacial predominantemente agregado (figura 3). Los valores de L(s) se incrementaron hasta $900 \mathrm{~m}, \mathrm{y}$ a partir de allí la curva asumió un comportamiento decreciente, lo que demuestra que el tamaño de los grupos dentro de la unidad de producción anual fue de hasta $900 \mathrm{~m}$ y después de esa escala los grupos son menos nítidos. Cuando se analizaron por separado, se añadieron los adultos maduros en etapa reproductiva, mientras que los juveniles no reproductivos tendían a la aleatoriedad en distancias de menos de $1.400 \mathrm{~m}$, a partir de los cuales fueron predominantemente agregados.

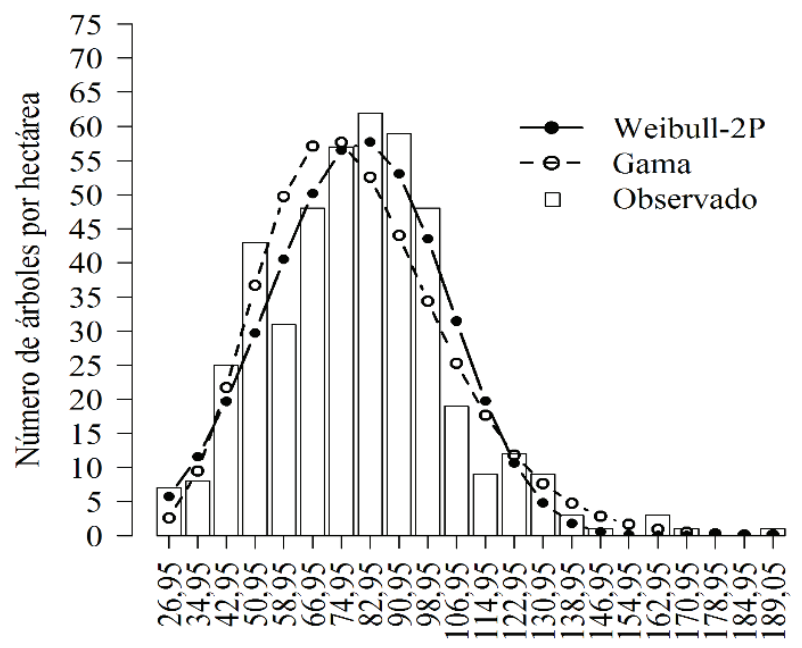

Centro de clase diamétrica $(\mathrm{cm})$

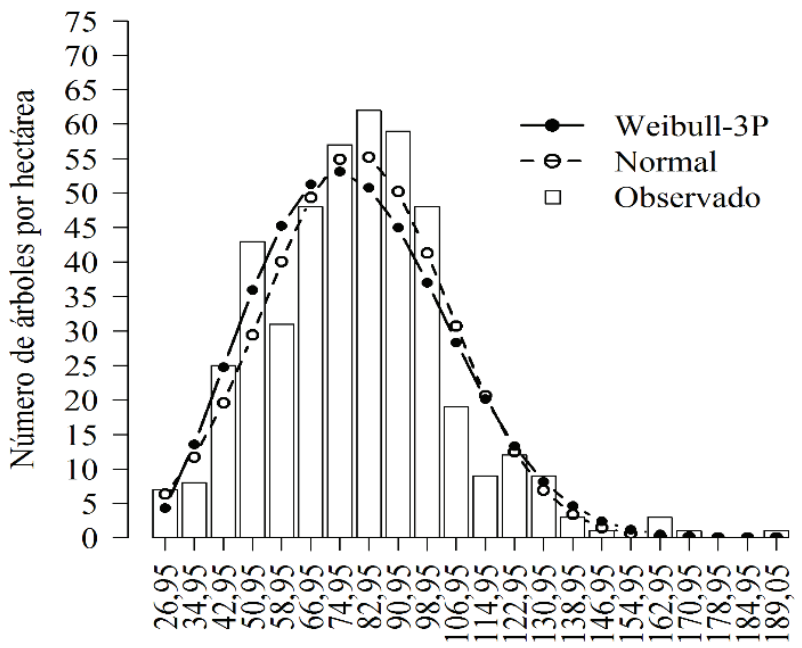

Centro de clase diamétrica $(\mathrm{cm})$

Figura 2. Distribución diamétrica observada y estimada por las cuatro principales funciones de densidad y probabilidad, utilizando métodos multivariados.

Diameter distribution observed and estimated by the four best functions of density and probability, using multivariate methods. 
Métodos de muestreo. Los procedimientos de muestreo aleatorio simple y sistemático tenían valores bajos de precisión, sin embargo, fueron más exactos que los métodos adaptativos (cuadro 1). Aunque en estos métodos se ha identificado una tendencia a sobreestimar el número total de árboles por hectárea, se encontró que la media estaba dentro de los intervalos de confianza estimados para muestreo aleatorio simple y muestreo sistemático (figura 4), lo que indica que estos métodos se pueden utilizar para estimar la densidad de individuos de B. excelsa.
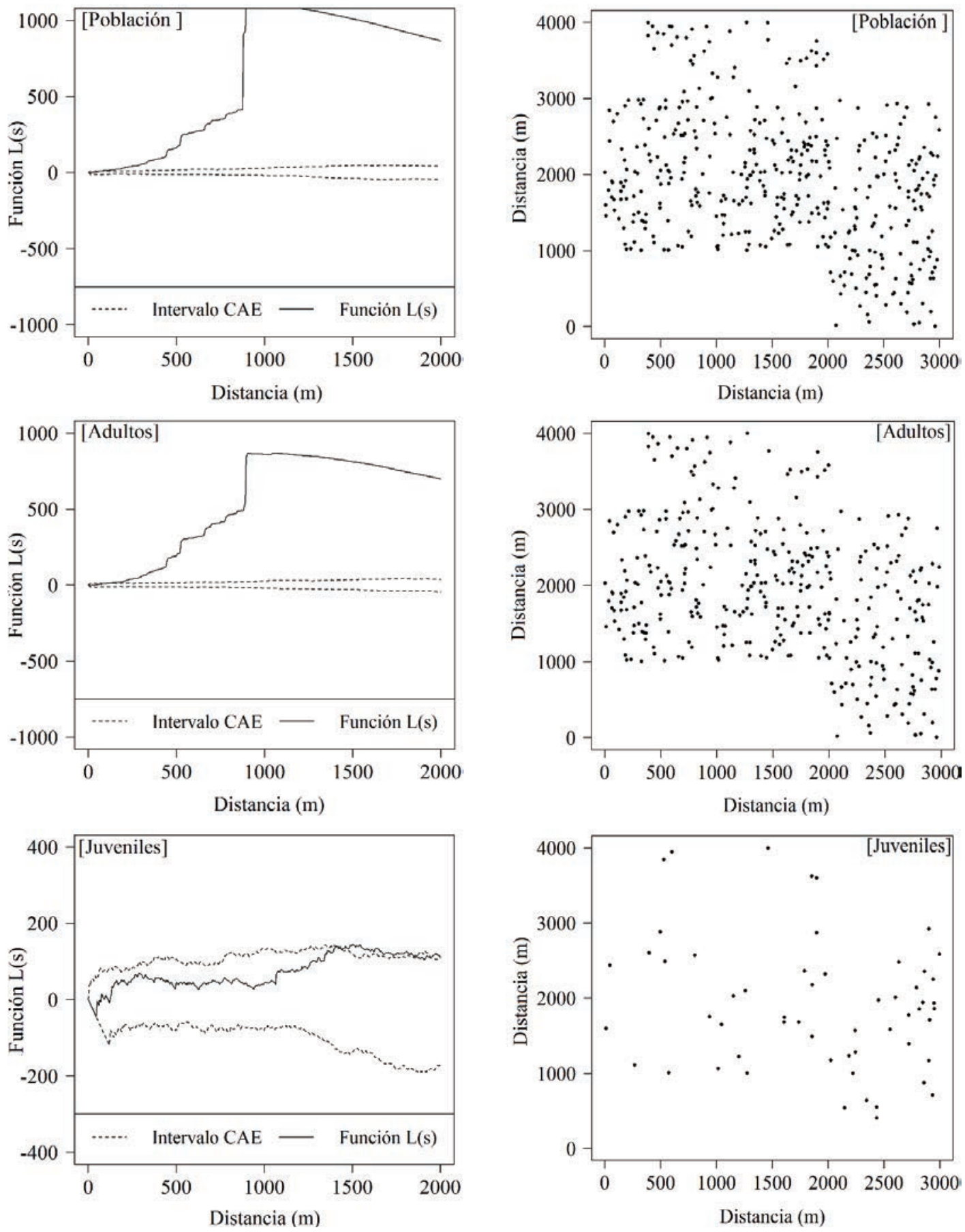

Figura 3. Patrón de distribución espacial de la población y las clases demográficas juveniles y adultas en el Bosque Nacional de Tapajós, estado de Pará. state of Pará.

Spatial distribution pattern for population and demographic classes, juveniles and adults, in the Tapajós National Forest, 
Cuadro 1. Promedio estimado de 30 simulaciones para los diferentes métodos de muestreo.

Average estimate of 30 simulations for different sampling methods.

\begin{tabular}{lccccccc}
\hline Alternativas de muestreo & $\bar{Y}$ & $S^{2}{ }_{\bar{Y}}$ & $S_{\bar{Y}}$ & $\pm E$ & $\pm E_{\%}$ & $\hat{Y}$ & $\mid E x a_{\%}$ \\
\hline MAS & 0,457 & 0,0032 & 0,0538 & 0,1057 & 23,3 & 457,1 & 9,95 \\
MS & 0,454 & 0,0025 & 0,0501 & 0,1035 & 22,9 & 454,2 & 5,35 \\
MAC-T & 0,276 & 0,0001 & 0,0110 & 0,0217 & 7,9 & 276,0 & 38,13 \\
MAC-M & 0,174 & 0,0001 & 0,0081 & 0,0159 & 9,2 & 174,1 & 60,96 \\
MAC-TF & 0,726 & 0,0001 & 0,0085 & 0,0167 & 2,3 & 720,6 & 62,71 \\
MAC-MF & 0,311 & 0,0001 & 0,0087 & 0,0170 & 5,4 & 311,2 & 30,22 \\
\hline
\end{tabular}

$\bar{Y}=$ media del número de individuos por hectárea; $S_{\bar{Y}}^{2}=$ varianza de media $S_{\bar{Y}}=$ error estándar de la media; $\pm E=$ error estándar absoluto; $\pm E_{\%}=$ de error de muestreo, en porcentaje; $\hat{Y}=$ total estimado; $\mid E x a_{\%}=$ precisión en módulo.
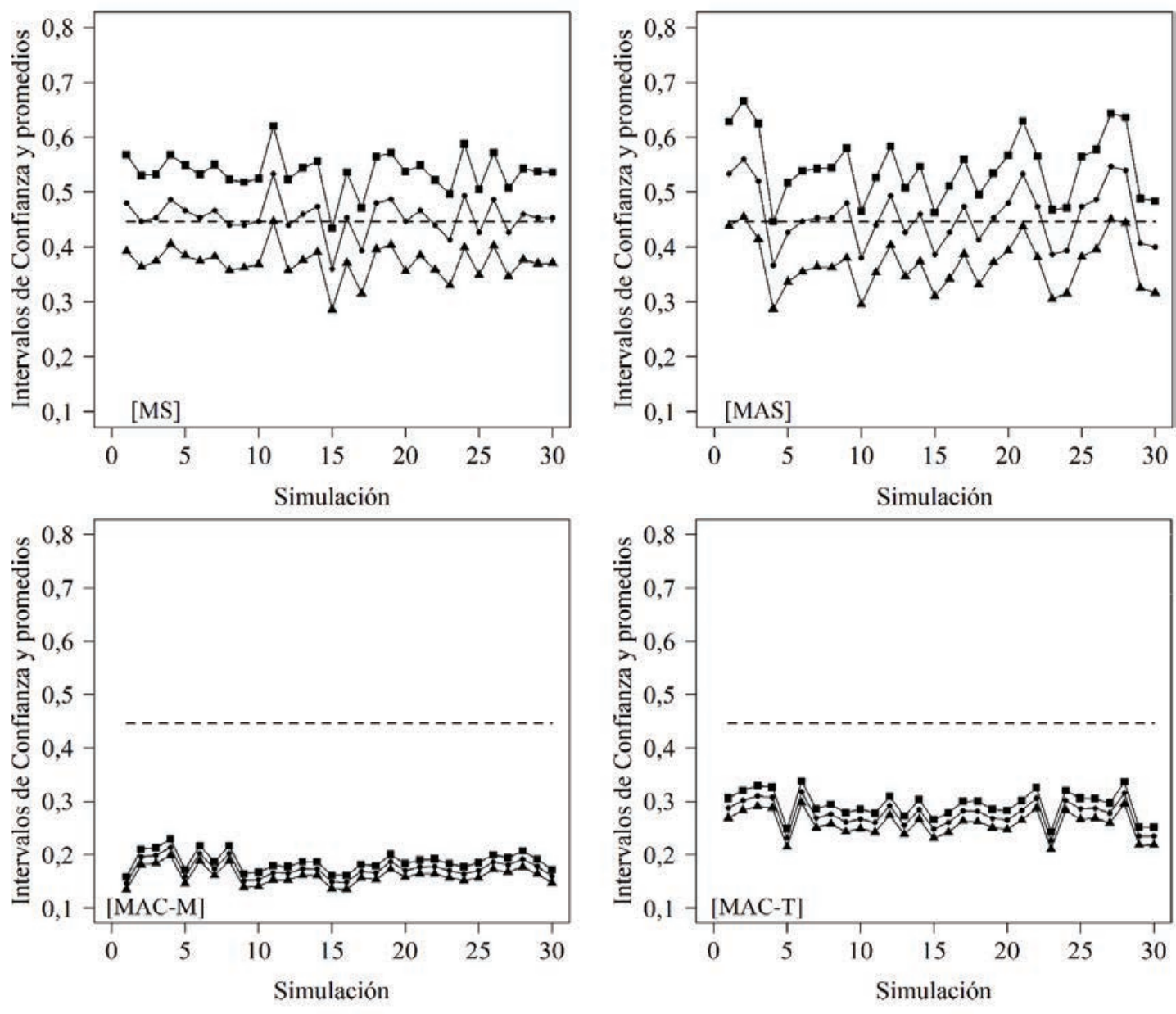

$\rightarrow$ Promedio Estimado -...-. Promedio Observado

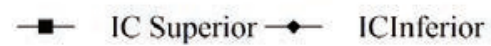

Figura 4. Número total estimado de las personas y los límites inferior y superior del intervalo de confianza para los métodos de muestreo. MAS = muestreo aleatorio simple; MS = muestreo sistemática; MAC-T= muestreo adaptativo por conglomerado tradicional por precariedad; y MAC-M = muestreo adaptativo por conglomerado modificado por precariedad.

Estimate of the total number of individuals and of the lower and upper limits of the confidence interval for the sampling method. Where: SSR = sampling simple random; SS = systematic sampling; ACS-C = adaptive cluster sampling traditional by randomization; ACS-M = adaptive sampling in modified cluster by randomization. 
Por otro lado, los métodos de adaptación fueron más precisas que muestreo aleatorio simple y muestreo sistemático, pero menos exactos y con una tendencia a subestimar el número total de árboles por hectárea (cuadro 1). Se encontró también que los intervalos de confianza para las medias estimadas por conglomerados adaptativos no cubrían la media real (figura 4), por lo que estos métodos son ineficaces para la cuantificación de los individuos B. excelsa. Además, se encontró que el uso de conglomerados modificados redujo las tasas de precisión y exactitud, mientras que el uso de bandas sistemáticas aumenta la precisión y reduce la exactitud cuando los conglomerados tradicionales son combinados. Esto se sucede debido al error de no muestreo, que no es explicado por el error estándar de la media. Cuando se combina con conglomerados modificados, el uso de bandas reduce tanto la precisión como la exactitud.

Cabe destacar, también que en todos los procedimientos de muestreo con la selección casual o sistemática de la muestra inicial, fueron observadas algunas unidades con la ausencia de individuos, denominadas como "parcelas cero". En el muestreo aleatorio simple y muestreo sistemático se observó una media de 537 y 535 unidades muestreadas con cero individuos, respectivamente. En MAC-T y MAC-M, se registraron 329 y 225 unidades de red con ausencia de individuos, respectivamente.

\section{DISCUSIÓN}

Estructura y distribución espacial. Bertholletia excelsa es una especie de árbol que se presenta con mayor frecuencia en las zonas de tierras no inundables de toda la región amazónica. En este estudio, la densidad total registrada (0,446 individuos ha-1) fue mayor que la estimada por Salomão (2009), en la meseta Aviso, al oeste de Pará, e inferior a la obtenida por Peres y Baider (1997), Peres et al. (2003), Wadt et al. (2005) y Scoles y Gribel (2011), que informaron variaciones desde 1,3 a 23,0 individuos $\mathrm{ha}^{-1}$ en la densidad de B. excelsa en diferentes lugares de la Amazonia. Esta amplia variación se debe a la gran variedad de diseños de muestra, los tamaños de las superficies de la muestra y la selección de las áreas investigadas, de B. excelsa o aleatoriamente (Wadt et al. 2005). Además, los efectos del cambio climático y edáfico, son también factores que influyen en esta variación en la densidad de las especies entre los distintos lugares de estudio.

La distribución de diámetro con una alta concentración de individuos en las clases intermedias y con unos pocos individuos en las clases de menor y mayor tamaño no es característico de las especies multietáneos. Sin embargo, debido al carácter heliófilo, longevo y dominante de la especie y la ausencia de alteraciones en la zona de estudio, este tipo de estructuras parece ser la más común para la especie, conforme demostraron Peres y Baider (1997), Peres et al. (2003) y Salomão (2009). Esta tendencia en la gama de diámetros puede ser descrita por las funciones de Weibull y Gamma, sirviendo como prueba de que los nuevos individuos se están estableciendo en la zona en pequeñas proporciones, lo que puede estar relacionado con la necesidad de condiciones específicas para la regeneración.

La mayoría de los estudios indican que la ausencia de individuos de B. excelsa en las clases inferiores a $60 \mathrm{~cm}$ es causada, principalmente, por el requerimiento de la luz para la especie, lo que resulta en el estancamiento del desarrollo del bosque tropical con dosel y del sub-bosque poco abierto (Mori y Prance 1990). Según Salomão (2009), el desarrollo completo solamente se produciría cuando hubiese alteraciones en la estructura del bosque que favorecen a una mayor incidencia de la luz en los estratos inferiores. En cuanto a Scoles y Gribel (2011), la estructura de la población es consecuencia de las influencias antropogénicas a las que son sometidas los bosques vírgenes y nativos, por lo que, si bien las poblaciones mayores de $B$. excelsa con bajos niveles de interferencia antropogénica tienen los árboles más antiguos y un menor número de semillas disponibles para las nuevas germinaciones, aquellos que tienen presencia constante de los seres humanos eran más jóvenes y reproductivos, es decir, con mayor número de individuos en las clases de menor diámetro. Por lo tanto, la regeneración de $B$. excelsa parece depender más de factores facilitadores de luz en el bosque que en las tasas de reclutamiento y la eficiencia de la dispersión de semillas naturales.

La extracción intensiva de los frutos de B. excelsa, a largo plazo, también puede ser un factor determinante en la estructura de la población de la especie. Según Peres et al. (2003), las poblaciones sometidas a altos o moderados niveles de colecta durante varias décadas carecen de árboles no reproductivos, mientras que las poblaciones cuyos antecedentes muestran la colección leve o reciente, o cualquier colección, tienden a tener un número significativo de individuos jóvenes. Por tanto, si no fueran adoptadas las políticas reguladoras para neutralizar los riesgos de una actividad no sostenible, el reclutamiento de jóvenes será insuficiente para mantener las poblaciones a largo plazo, terminando por sucumbir por medio del proceso de la senescencia y el proceso de colapso demográfico.

En los bosques tropicales, la estructura espacial de las especies de árboles está altamente determinada por los síndromes de dispersión de semillas y por la aparición de micrositios favorables para el desarrollo de los individuos (Seidler y Plotkin 2006), por lo que las poblaciones con frutos dispersos de forma zoocoria y barocoria tienden a presentar patrones espaciales agregados (Nyiramana et al. 2011), conforme lo observado para la población y los individuos reproductivamente maduros. Peres y Baider (1997), Wadt et al. (2005) y Salomão (2009) encontraron a través del índice con base en unidades muestreadas, patrones predominantemente agregados en los individuos de B. excelsa. Esto ocurrió porque, normalmente, estos síndromes limitan la distribución de plántulas resultando en altas tasas de germinación y reclutamiento en zonas cer- 
canas al árbol origen, mientras que en otros lugares estos procesos son más pequeños y aleatorios.

Aunque se espera que hay conglomerados de individuos jóvenes no reproductivos entre 10 y 300 metros del límite de proyección de las copas de los árboles, se encontró que estos grupos estaban presentes solo en escalas de distancia mayores de $1.400 \mathrm{~m}$, lo que determina el predominio de un patrón aleatorio en las distancias menores de $1.400 \mathrm{~m}$. Este hecho parece ser un reflejo de la dinámica de los individuos con diámetros inferiores a $20 \mathrm{~cm}$, ya que en estas clases hay una capacidad mínima de respuestas a las condiciones bióticas (es decir, la competencia intra e interespecífica entre especies, tipo de dispersión de los propágulos) y abióticas (es decir, la disponibilidad de agua, los nutrientes del suelo y la luz), que asociadas a la presencia de herbívoros y patógenos, considerada la principal causa de mortalidad entre las plántulas y árboles jóvenes, puede estar afectando la densidad de B. excelsa, a fin de promover una distribución aleatoria.

Métodos de muestreo. Los inventarios forestales se realizan comúnmente para obtener estimaciones exactas a un bajo costo y tiempo, pero cuando se trata de inventarios no maderables las estimaciones por métodos tradicionalmente aplicados a los productos de madera son deficientes y muchas veces resultan en altas intensidades de la muestra (Bruzinga et al. 2014). En este estudio, el muestreo aleatorio simple y sistemático fueron los procedimientos que han demostrado los mejores índices de precisión; sin embargo, eran los que tenían errores de muestreo de más de $10 \%$, que se define como el límite máximo aceptable para la variable considerada en el inventario forestal. Una de las razones probables para este comportamiento fue el elevado número de parcelas cero en este estudio, lo que llevó a los aumentos de las estimaciones de la varianza media, del error estándar de la media y de los errores de muestreo.

Una de las soluciones tradicionalmente adoptadas en este caso es el cambio de tamaño de la muestra para obtener un error de la muestra por debajo de $10 \%$ (Shiver y Borders 1996) para producir, respectivamente, el muestreo de intensidades de 42 y $40 \%$ de la superficie total, el cual cambia de forma indirecta los costes finales del inventario por muestreo aleatorio simple y muestreo sistemático, respectivamente, a 180 y 166,6 \% del valor inicial. Sin embargo, se encontró que la media obtenida para la encuesta de prospección estaba dentro de los intervalos de confianza estimados para muestreo aleatorio simple y muestreo sistemático, que muestra que en 95 de 100 muestras aleatorias o sistemáticas la media de la población estaba dentro del intervalo de confianza establecido. De ese modo, teniendo en cuenta la exactitud de los valores e intervalos de confianza como criterios para evaluar la calidad de los inventarios no maderables, se encontró que los métodos tradicionales asociados a la intensidad de muestreo del $15 \%$ son eficaces para estimar el número de individuos por hectárea de B. excelsa, a pesar de que hay una ligera tendencia de sobreestimación. En la práctica, la aplicación de un muestreo sistemático es más simple, menos costoso, debido a la distribución uniforme de las parcelas en el campo, siendo fácil de encontrar, como previsto por la re-evaluación y seguimiento (Krebs 1999).

Por el contrario, el análisis del muestreo adaptativo por conglomerado (MAC) demostró ser más preciso, pero menos exacto que los procedimientos tradicionales, que los hacían inviables para cuantificar las poblaciones de B. excelsa. Para estas alternativas de muestreo, el uso de intervalos de confianza corroboran las estimaciones de precisión y exactitud, dado que el promedio real no estaba dentro de los intervalos de confianza estimados para cualquiera de las 30 simulaciones. Se encontró también que los procedimientos de adaptación mostraron una clara tendencia a subestimar el número total de individuos, a excepción de procedimiento MAC-MF. Este hecho puede explicarse por el pequeño tamaño de las redes presentes en el área de toma de muestras y el bajo número de individuos presentes en cada unidad de la muestra, generando pesos $\left(w_{i}\right)$ pequeños y confiriendo este comportamiento a la estimación de Hansen-Hurwitz modificado para la media (Soares et al. 2009).

La eficacia de los procedimientos del muestreo adaptativo por conglomerados se puede aumentar debido a la configuración de la vecindad y la elección de los criterios de inclusión, debiéndose considerar no solo la presencia del individuo en unidades de red, sino también otras condiciones de inclusión. Sin embargo, se debe tener cuidado de que estos criterios no sean demasiado bajos ni demasiado altos, ya que según Brown (2003), si se establece los criterios de inclusión con un valor muy bajo, el tamaño final de la muestra puede ser demasiado grande, una vez que las redes que serán formadas puedan englobar muchas parcelas. De lo contrario, si el valor de los criterios de inclusión es muy alto, pocas parcelas serán incluidas en el proceso de muestreo (Soares et al. 2009).

La escala de la agregación y el tamaño y forma de las parcelas fueron probablemente los factores que más influenciaran los estimadores de muestreo adaptativo por conglomerados, en virtud de las mayores distancias entre individuos de B. excelsa (Krebs 1999). Por ejemplo, Soares et al. (2009) en un bosque simulado de 90 hectáreas, subdividido en 100 unidades de muestra de $9.000 \mathrm{~m}^{2}$ cada una, concluirían de que a bajas intensidades del muestreo adaptativo por conglomerado con un criterio de inclusión igual a un árbol, era más eficiente, pero si se utilizan parcelas similares a las simuladas los resultados de este estudio probablemente no serían los mismos. Por otra parte, Bruzinga et al. (2014), en un área de 36,5 hectáreas dividida en unidades de muestreo de $400 \mathrm{~m}^{2}$, llegaron a la conclusión de que el muestreo adaptativo por conglomerado con condición de inclusión igual a dos individuos, era el método más preciso para obtener la densidad de Caryocar brasiliensis Camb. (pequi), pero la escala de agregación de individuos de pequi era mucho más grande (Bruzinga et al. 2013) que las encontrados para los individuos de B. excelsa. 
Cabe destacar que para muchas especies no maderables, para incluir $B$. excelsa, no hay técnicas de muestreo específicos que sean impuestos por los organismos públicos (IBAMA, Departamentos de Medio Ambiente) en el desarrollo de los procedimientos básicos para la gestión sostenible de los productos secundarios de estas especies. En la Amazonia brasileña, se tiene conocimiento de unas pocas resoluciones e instrucciones normativas (Instrucción Normativa $N^{\circ} 01$ de 11/02/2008, Instrucción Normativa $\mathrm{N}^{\circ} 03$ de 10/09/2007, Resolución COEMA No 13 de 30/07/2009 y Instrucción Normativa nº 09 de 26/08/2011) que establecen procedimientos para la extracción y comercialización de productos forestales no madereros (por ejemplo, Heteropsis sp. y Philodendron sp.), que se basan en estudios que muestran la intensidad y los métodos adecuados para cuantificar este tipo de especie. Por otra parte, en algunas de estas normas se consideran los errores de muestreo superior a $15 \%$, según lo recomendado por Silva (2014), al analizar los métodos de muestreo de Heteropsis sp. En este estudio, se recomienda el uso del intervalo de confianza para la estimación de la media por procedimientos de muestreo como criterio para evaluar la calidad del inventario forestal, y los esfuerzos adicionales con el fin de investigar el efecto del tamaño y forma de las parcelas y la escala de agregación, y sus combinaciones sobre la eficiencia de los estimadores del muestreo adaptativo por conglomerado.

\section{CONCLUSIONES}

La población y los adultos reproductivos muestra un patrón espacial agregado, mientras que el patrón de los individuos jóvenes no reproductivos es predominantemente aleatorio.

El uso del muestreo adaptativo por conglomerados no es eficiente para estimar el número de individuos por hectárea. Por otro lado, los métodos de muestreo aleatorio simple y sistemático pueden utilizarse en la cuantificación de los individuos de B. excelsa, puesto que consideran el intervalo de confianza como parámetro para la evaluación de la calidad del inventario forestal.

\section{AGRADECIMIENTOS}

Agradecemos a la CAPES, por la beca al primer autor. A la COOMFLONA por poner a disposición los datos.

\section{REFERENCIAS}

Alechandre A, F Brown, CA Campos, K Azevedo, T Melo. 2007. Cartilha de mapeamento de espécies florestais: um guia simplificado para a sistematização em planilha eletrônica de mapa de campo de espécies florestais. Rio Branco, Brasil. UFAC. 22 p.

Brown JA. 2003. Designing an efficient adaptive cluster sample. Environmental and Ecological Statistics 10(1): 95-105.

Bruzinga JS, MLR Oliveira, ELM Machado, HG Leite, IM Perei- ra, GS Nogueira. 2013. Distribuição espacial de indivíduos adultos de pequi. Scientia Forestalis 41(98): 249-256.

Bruzinga JS, MLR Oliveira, GS Nogueira, IM Pereira, HG Leite, ELM Machado. 2014. Métodos de amostragem para quantificar indivíduos adultos do pequi Caryocar brasiliense Cambess. Ciência Rural 44(8): 1341-1347.

Christman MC. 2003. Adaptive Two-Stage One-per-Stratum Sampling. Envorinmental and Ecological Statistics 10(1): 43-60.

Cochran WG. 1977. Sampling tecniques. New York, U.S. John Wiley. $428 \mathrm{p}$.

Hansen MM, WN Hurwitz. 1943. On the theory of sampling from finite populations. Annals of Mathematical Statistics 14(4): 333-362.

Krebs CJ. 1999. Ecological methodology. New York, U.S. Addison Wesley Longman. 620 p.

Mori SA, GT Prance. 1990. Taxonomy, ecology and economic botany of the Brazil nut (Bertholletia excelsa Humb. et Bonpl.: Lecythidaceae). Advances in Economic Botany 8: 130-150.

Nyiramana A, I Mendoza, BA Kaplin, PM Forget. 2011. Evidence for Seed Dispersal by Rodents in Tropical Montane Forest in Africa. Biotropica 43(6): 654-657.

Peres CA, C Baider, PA Zuidema, LHO Wadt, KA Kainer, DAP Gomes-Silva, RP Salomão, LL Simões, ERN Franciosi, FC Valverde, R Gribel, GHJ Shepard, M Kanashiro, P Coventry, DW Yu, AR Watkinson, RP Freckleton. 2003. Demographic threats to the sustainability of Brazil nut exploitation. Science 302: 2112-2114.

Peres CA, Baider C. 1997. Seed dispersal, spatial distribution and population structure of Brazil nut trees (Bertholletia excelsa) in southeastern Amazonia. Journal of Tropical Ecology 13: 595-616.

Ripley BD. 1979. Tests of randomness for spatial point patterns. Journal of the Royal Statistical Society. Serie B (Methodological) 41(3): 368-374.

Salomão RP. 2009. Densidade, estrutura e distribuição espacial de castanheira-do-brasil (Bertholletia excelsa H. et B.) em dois platôs de floresta ombrófila densa na Amazônia setentrional brasileira. Boletim do Museu Paraense Emílio Goeldi 4(1): 11-25.

Scoles R, R Gribel. 2011. Population Structure of Brazil Nut (Bertholletia excelsa, Lecythidaceae) Stands in Two Areas with Different Occupation Histories in the Brazilian Amazon. Human Ecology 39(4): 455-464.

Seidler TG, JB Plotkin. 2006. Seed dispersal and spatial patterns in tropical trees. PLoS Biology 4(11): 2132-2137.

Shiver BD, B Borders. 1996. Sampling techniques for forest resource inventory. New York, U.S. John Wiley. 356 p.

Silva CK. 2014. Potencial produtivo e de manejo de dois produtos florestais não madeireiros no contexto Amazônico - o cipó titica (Heteropsis spp.) e o óleo de copaíba (Copaifera spp.). Tese Doutorado em Ciência Florestal. Piracicaba, Brasil. Faculdade de Ciência Florestal, Universidade de São Paulo. 145 p.

Smith DR, JA Brown, NCH Lo. 2004. Application of adaptive cluster sampling to biological populations. In Thompson WL eds. Sampling Rare Populations. Washington, U.S. Island Press. p. 77-122.

Soares CPB, CM Rodello, ALS Souza, HG Leite, VP Soares, GFS Silva. 2009. Comparação entre os procedimentos de amostragem para espécies florestais raras e padrão de dis- 
tribuição agregado. Floresta 33(3): 545-553.

Souza DR, AL Souza, JRV Gama, HG Leite. 2003. Emprego de análise multivariada para estratificação vertical de florestas inequiâneas. Árvore 27(1): 59-63.

Thompson SK. 1990. Adaptive cluster sampling. Journal of the American Statistical Associoation 85(412): 1050-1059.

Thompson SK, GAF Seber.1996. Adaptive sampling. New York, U.S. John Wiley. 265 p.

Tonini H. 2013. Amostragem para a estimativa de produção de sementes de castanheira-do-brasil em floresta nativa. Pesquisa Agropecuária Brasileira 48(5): 519-527.

Wadt LHO, Kainer KA, DAP Gomes-Silva. 2005. Population structure and nut yield of a Bertholletia excelsa stand in
Southwestern Amazonia. Forest Ecology and Management 211(3): 371-384.

Veloso HP, ALR Rangel Filho, JCA Lima. 1991. Classificação da vegetação brasileira a um sistema universal. Rio de Janeiro, Brasil. Fundação Instituto Brasileiro de Geografia e Estatística. 123 p.

Vieira DS. 2015. Padrão espacial de espécies arbóreas no Baixo Rio Tapajós. Dissertação Mestrado em Ciência Florestal. Diamantina, Brasil. Departamento de Engenharia Florestal, Universidade dos Vales do Jequitinhonha e Mucuri. 99 p.

Wong JLG, K Thornber, N Baket. 2001. The biometrics of nontimber forest product resource assessment: a review of current methodology. Roma, Italian. FAO. 109 p.

Recibido: 29.08.16

Aceptado: 01.12.16 
